\title{
Laparoscopic Partial Adrenalectomy: Surgical Technique and Outcome
}

\author{
Oh Seok Ko, Ji Yong Kim, Hyung Jin Kim, Young Beom Jeong
}

\begin{abstract}
Department of Urology, Chonbuk National University Medical School, Research Institute of Clinical Medicine of Chonbuk National University-Biomedical Research Institute of Chonbuk

National University Hospital, Jeonju, Korea
\end{abstract}

Purpose: To examine the perioperative surgical and long-term outcomes of laparoscopic partial adrenalectomy (LPA) by comparing the results of laparoscopic total adrenalectomy (LTA)

Materials and Methods: A total of 132 transperitoneal laparoscopic adrenalectomies were performed for adrenal gland tumor between May 2006 and April 2019. All surgeries were performed by a single surgeon. Seventy patients underwent LTAs and 54 underwent LPAs. The data were collected retrospectively through review of the medical charts. We compared the perioperative and long-term outcomes between the 2 surgical methods.

Results: A total of 70 patients, including 4 patients with bilateral tumors, underwent LTA. In contrast, 54 patients, including 4 patients with bilateral masses, underwent LPA. There were no differences between the 2 groups with regard to mean age at presentation, mean tumor size, or postoperative stay. However, the mean operating time was significantly shorter in the LPA group than that of the LTA group. The mean estimated blood loss in the LPA group was significantly higher than that in the LTA group. However, none of the LPA patients required blood transfusion. The biochemical markers and laboratory values normalized postoperatively in all patients with functional adrenal tumors. There was no local recurrence during the follow-up period.

Conclusions: Our data demonstrate that the surgical outcomes and perioperative complications in LPA group are similar to those of LTA. When LPA is performed for small adrenal lesions, most patients remain steroid independent, as well as recurrence-free at long-term follow-up. (Korean J Urol Oncol 2019;17:103-109)

Key Words: Adrenal glands · Partial adrenalectomy • Laparoscopy • Outcome $\cdot$ Recurrence

\section{INTRODUCTION}

Total adrenalectomy (TA) has classically been the standard surgical therapy for adrenal tumors, regardless of hormone sta-

Received July 16, 2019,

Accepted July 30, 2019

Corresponding Author: Young Beom Jeong

Department of Urology, Chonbuk National University Medical

School, Research Institute of Clinical Medicine of Chonbuk

National University-Biomedical Research Institute of Chonbuk

National University Hospital, 20 Geonji-ro, Deokjin-gu, Jeonju

54907, Korea

E-mail: ybjeong@jbnu.ac.kr

Tel: +82-63-250-1911, Fax: +82-63-250-1564

ORCID code: https://orcid.org/0000-0002-4054-3072 tus or tumor size. However, TA increases the risk of adrenal insufficiency and the need for life-long steroid replacement therapy following the operation in case of bilateral tumors. These factors can ultimately decrease health-related quality of life. ${ }^{1}$ Despite adequate steroid replacement, Addisonian crisis can occur in up to $25 \%-33 \%$ of patients who undergo bilateral adrenalectomy. ${ }^{2,3}$ Because adrenal glands are paired organs, an organ preservation approach, similar to that applied in the treatment of renal tumors, can be used. Organ preservation is being increasingly practiced; however, there is no strong evidence that maximal adrenal preservation is paramount. Most patients who undergo unilateral TA have sufficient hormonal reserve from the functioning, contralateral adrenal gland. Of course, there is

This is an Open Access article distributed under the terms of the Creative Commons Attribution Non-Commercial License (http://creativecommons.org/licenses/by-nc/4.0/) which permits unrestricted non-commercial use, distribution, and reproduction in any medium, provided the original work is properly cited. 2019 (C) Copyright The Korean Urological Oncology Society and The Korean Prostate Society. All Rights Reserved. 
an exception in Cushing's syndrome because of contralateral adrenal atrophic change. Regardless, some investigators have found that, after unilateral adrenalectomy, patients with one functioning adrenal gland often have suboptimal hormonal responses to stress postoperatively., ${ }^{3,4}$ In addition, there are a number of potential threats to the contralateral adrenal gland throughout an individual's life. ${ }^{5,6}$ For instance, this organ is susceptible to idiopathic, infectious, infiltrative, neoplastic, traumatic, and drug-related adrenal insufficiency. Therefore, maximal adrenal preservation is preferred given the low surgical risks and low possibility for recurrence associated with adrenal preserving surgery, as well as the potential danger of adrenal insufficiency with bilateral resection.

Endocrinologists and urologists encounter nearly all cases of atrophy of the contralateral adrenal gland in cases of unilateral Cushing's adenoma. Given the hazards and potential problems associated with adrenal insufficiency, some researchers recommend adrenal preserving surgery in small, unilateral adrenal incidentaloma, even in patients without a familial syndrome., There have been some reports for adrenal cortical sparing surgery to reduce necessity of steroid replacement therapy. ${ }^{9-12}$

Therefore, we evaluated the long-term outcomes of laparoscopic partial adrenalectomy (LPA) compared with those of laparoscopic total adrenalectomy (LTA).

\section{MATERIALS AND METHODS}

This study was performed in accordance with the World Medical Association's Declaration of Helsinki-Ethical Principles for Medical Research Involving Human Subjects. This retrospective protocol was approved by the Institutional Review Board of the Chonbuk National University Hospital (approval number: 2019-05-074-001). Between May 2006 and April 2019, 124 patients underwent laparoscopic adrenalectomy performed by a single surgeon. The mean patient age was $49.7 \pm 12.5$ years in the LTA group and $50.6 \pm 9.4$ years in the LPA group. Biochemically active tumors and nonfunctioning tumors with proven growth during the periodic follow-up were indicated for adrenalectomy. For patients with Conn's adenoma, adrenalectomy was performed if a unilateral tumor was identified by computed tomography (CT) or magnetic resonance imaging (MRI). However, cases of bilateral adrenal hyperplasia in which there was not a definitive nodule or mass on imaging studies were excluded from this retrospective study. LTA was performed in 70 patients. LTA group included 3 subjects with bilateral pheochromocytoma and 1 patient with bilateral macronodular hyperplasia. Fifty-four patients including 4 bilateral cases underwent LPA. Simultaneous, bilateral LPAs were performed in 3 patients with bilateral Conn's adenomas and in a huge bilateral pheochromocytoma. Overall, 74 tumor-bearing adrenal glands were completely removed, while 58 adrenal glands were partially resected. Four patients with bilateral pheochromocytoma had inherited multiple endocrine neoplasms (MEN) type 2A. Patient characteristics are presented in Table 1.

Patients with pheochromocytoma were pretreated with oral doxazocin $(4 \mathrm{mg})$ at least 2-3 weeks prior to the surgery. In cases of Conn's adenoma, potassium-sparing diuretics and oral potassium replacement therapy were administered preoperatively by the Department of Endocrinology. Similarly, in patients with Cushing's syndrome, perioperative corticosteroid treatment was done at the discretion of the endocrinology service. In most cases of functioning adrenal tumors, postoperative hormonal status was assessed, and additional hormonal and antihypertensive drugs were titrated as needed. In order to evaluate long-term outcomes, we retrospectively reviewed the medical chart and contacted by telephone some patients lost to clinical follow-up. We compared the perioperative and long-term outcomes between the 2 surgical methods.

Statistical analyses were conducted by an expert in medical statistics. The independent-samples t-test and Pearson chi-square test with IBM SPSS ver. 18.0 (IBM Co., Armonk, NY, USA) were used. P-values $<0.05$ were considered statisti-

Table 1. Comparison of baseline patient characteristics between LTA and LPA

\begin{tabular}{lccc}
\hline \multicolumn{1}{c}{ Characteristic } & LTA & LPA & p-value \\
\hline No. of patients & 70 & 54 & \\
Age at presentation (yr) & $49.7 \pm 12.5$ & $50.6 \pm 9.4$ & 0.563 \\
Mean tumor size $(\mathrm{cm})$ & $3.37 \pm 1.86$ & $3.27 \pm 1.65$ & 0.625 \\
Body mass index $\left(\mathrm{kg} / \mathrm{m}^{2}\right)$ & $24.2 \pm 2.6$ & $25.5 \pm 2.3$ & 0.896 \\
Tumor site (n) & & & \\
$\quad$ Right:left & $28: 38$ & $22: 28$ & 0.034 \\
Bilateral* & 4 & 4 & \\
\hline
\end{tabular}

Values are presented as mean \pm standard deviation unless otherwise indicated.

A total of 132 adrenalectomies were performed.

LTA: laparoscopic total adrenalectomy, LPA: laparoscopic partial adrenalectomy.

* Bilateral cases were excluded in comparison of perioperative outcome between the 2 groups. 
cally significant.

\section{Surgical Procedures for LPA}

Under general anesthesia, the patient was placed in the modified semilateral position with the lesion side elevated by approximately $60^{\circ}$. Pneumoperitoneum was established using the open Hasson technique. Typically, we used two 10-mm working ports, one 5-mm working port, and one 12-mm camera port for each side procedure. The first 12-mm camera port was inserted at the lateral border of the rectus abdominis muscle at the level of the umbilicus. Two 10-mm ports were then placed along the midclavicular line and anterior axillary line so that the 2 trocars with camera ports make an equilateral triangular configuration.

A third 5-mm subcostal trocar was inserted in the subcostal area in order to retract the liver or spleen. After pneumoperitoneum was established with $15 \mathrm{mmHg}$ of $\mathrm{CO}_{2}$, a peritoneal incision was made along the white line of Toldt to mobilize the colon. After incising Gerota's fascia, we approached the upper pole of the kidney and then dissected the loose areolar tissue surrounding the adrenal vein. The main adrenal vein was identified, and further dissection of the tumor surrounding tissue was continued. Adrenal vein isolation was not necessary when the tumor was located at the edge of the adrenal limbs. Adrenal gland manipulation was carefully performed using a blunt monopolar dissector to minimize injury to the normal adrenal tissue. Hem-o-lok clips were applied between the tumor and normal cortex for bleeding control, and then the adrenal tumor was resected along normal adrenal tissue (Fig. 1).

\section{RESULTS}

Seventy patients underwent unilateral LTA and 4 patients underwent bilateral LTA. Fifty-four patients, including 4 with bilateral tumors, underwent LPA. There were no mortalities in our consecutive series of 132 laparoscopic adrenalectomies. There were no differences between the 2 groups with regard to mean age at presentation, mean tumor size and BMI (Table 1). However, the mean operating time was significantly shorter in the LPA group $(168.7 \pm 63.7$ minutes vs. $123.5 \pm 51.3$ minutes, $\mathrm{p}<0.001)$. On the other hand, the mean estimated blood loss was significantly higher in the LPA group than that of the LTA group $(39.3 \pm 48.6 \mathrm{~mL}$ vs. $62.3 \pm 30.6 \mathrm{~mL}, \mathrm{p}=0.021)$. Two conversions occurred due to intraoperative bleeding and technical difficulty in the LTA group. The length of postoperative stay

Table 2. Comparison of perioperative surgical outcome by groups

\begin{tabular}{lccc}
\hline \multicolumn{1}{c}{ Parameter } & LTA & LPA & p-value \\
\hline $\begin{array}{l}\text { Mean operative time } \\
\text { (min) }\end{array}$ & $168.7 \pm 63.7$ & $123.5 \pm 51.3$ & 0.001 \\
Mean EBL (mL) & $39.3 \pm 48.6$ & $62.3 \pm 30.6$ & 0.021 \\
Postoperative stay (day) & $6.01 \pm 2.23$ & $6.05 \pm 1.96$ & 0.847 \\
Open conversion* (n) & 2 & 0 & \\
\hline
\end{tabular}

Values are presented as mean \pm standard deviation unless otherwise indicated.

LTA: laparoscopic total adrenalectomy, LPA: laparoscopic partial adrenalectomy, EBL: estimated blood loss.

Open conversion and bilateral cases were excluded in comparison of perioperative surgical outcome between the 2 groups.
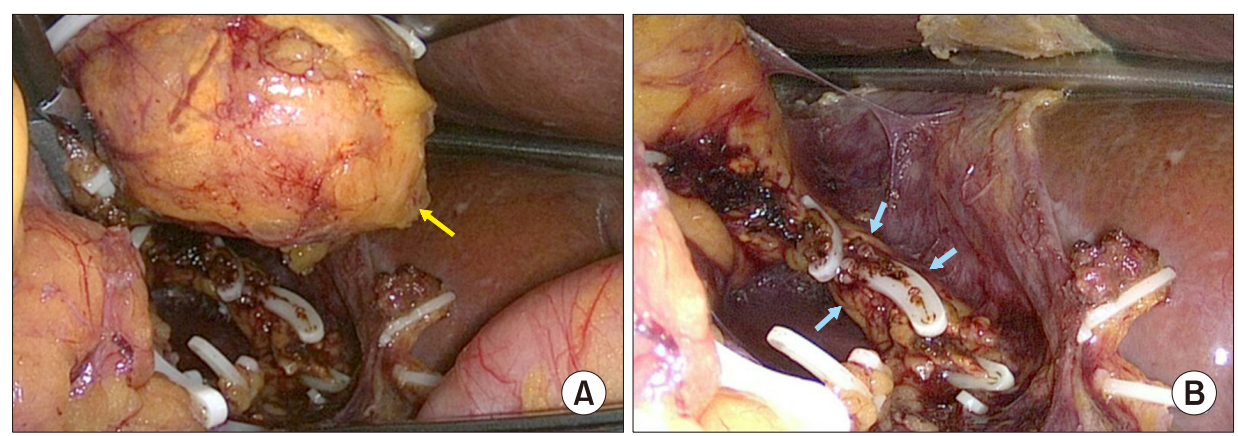

Fig. 1. Intraoperative views of transperitoneal laparoscopic right partial adrenalectomy. (A) Resected adrenal mass (white arrow). (B) Remnant adrenal tissue in the subhepatic space after completion of laparoscopic partial adrenalectomy. Good hemostasis was achieved using Hem-o-lok clips (Weck Surgical Instruments, Teleflex Medical, Durham, NC, USA) with minimal dissection of the tissues around the remnant adrenal tissue (black arrows). 
Table 3. Long-term functional outcomes by groups

\begin{tabular}{lrr}
\hline \multicolumn{1}{c}{ Parameter } & LTA & LPA \\
\hline Follow-up (mo), mean \pm SD & $50.5 \pm 43.6$ & $49.2 \pm 39.5$ \\
Blood pressure in CA & 75 & 73 \\
$\quad$ Normalized (\%) & 25 & 27 \\
$\quad$ Sustained, needed medication (\%) & 100 & 100 \\
Normalization of serum potassium level and renin-aldosterone ratio in CA (\%) & 100 \\
Normalization of plasma or urinary metanephrine level in pheochromocytoma (\%) & $6 / 6(100)$ & $8 / 8(100)$ \\
Temporary steroid replacement in CS (\%) & $4 / 4(100)$ & $0 / 4(0)$ \\
Permanent steroid replacement in bilateral cases (\%) & - & - \\
Local recurrence* & &
\end{tabular}

In cases of CS, perioperative steroid replacement was performed by endocrinologists.

LTA: laparoscopic total adrenalectomy, LPA: laparoscopic partial adrenalectomy, SD: standard deviation, CA: Conn's adenoma, CS: Cushing's syndrome.

*Primary and secondary cancer cases were excluded.

Table 4. Surgical indications by groups

\begin{tabular}{lcc}
\hline \multicolumn{1}{c}{ Diagnosis } & $\begin{array}{c}\text { LTA } \\
(\mathrm{n}=74)\end{array}$ & $\begin{array}{c}\text { LPA } \\
(\mathrm{n}=58)\end{array}$ \\
\hline Pheochromocytoma & 17 & 16 \\
NAA & 14 & 13 \\
Conn's adenoma & 16 & 11 \\
Cushing's syndrome $^{\text {Adrenal cyst* }}$ & 6 & 8 \\
Metastatic tumor $^{\dagger}$ & 2 & 6 \\
Primary adrenal carcinoma $_{\text {Neural tumor }}$ & 8 & $1^{\ddagger}$ \\
Borderline malignant tumor $_{\text {Adenomatoid tumor }}$ & 3 & $1^{\S}$ \\
Malignant pheochromocytoma & 1 & 2 \\
\hline
\end{tabular}

A total of 132 adrenalectomies were performed in 124 patients.

LTA: laparoscopic total adrenalectomy, LPA: laparoscopic partial adrenalectomy, NAA: nonfunctioning adrenocortical adenoma.

*Includes pseudocyst, cyst with myelolipomatous metaplasia, and epithelial and endothelial (vascular) cysts. ${ }^{\dagger}$ Includes 5 metastatic tumors from lung cancer, 3 from renal cell carcinoma and 1 breast cancer. ${ }^{\ddagger} \mathrm{LPA}$ was done, but there was no local recurrence for 10 months. ${ }^{\S} \mathrm{LPA}$ was performed under-diagnosis of Cushing's syndrome, but final pathologic result was primary adrenocortical carcinoma. In this case, there has been no recurrence for 24 months of follow-up.

was similar in the 2 groups $(6.01 \pm 2.23$ days vs. $6.05 \pm 1.96$ days, $\mathrm{p}=0.847$ ) (Table 2). The mean follow-up period was $50.5 \pm 43.6$ months in the LTA group and $49.2 \pm 39.5$ months in the LPA group, respectively. Hypokalemia and renin-aldosterone ratio were normalized in all patients with Conn's adenoma after the surgery. However, about $25 \%$ of patients with primary
Table 5. Perioperative complications

\begin{tabular}{lcc}
\hline \multicolumn{1}{c}{ Complication } & LTA & LPA \\
\hline Intraoperative & 3 & 2 \\
Liver or spleen tears & 1 & 0 \\
Intraoperative bleeding $>200 \mathrm{~mL}$ & & \\
Postoperative & 1 & 1 \\
Atelectasis & 1 & 0 \\
Mechanical ileus & 1 & 0 \\
Intraabdominal abscess formation & 4 & 5 \\
Delayed healing of trocar site & 1 & 0 \\
Adrenal crisis &
\end{tabular}

LTA: laparoscopic total adrenalectomy, LPA: laparoscopic partial adrenalectomy.

aldosteronism still required antihypertensive medications in both groups. All patients with functional adrenal masses such as pheochromocytoma, Conn's adenoma, and Cushing's syndrome demonstrated postoperative normalization of biochemical laboratory results. Four patients who underwent simultaneous bilateral LTA continued corticosteroid therapy postoperatively. In contrast, 4 bilateral cases in the LPA group received steroid replacement temporarily, but all of them were able to cease the hormone supplement within 6 months after the surgery. There was no local recurrence during follow-up in both groups (Table 3 ). The most common surgical indication was pheochromocytoma, followed by NAA, Conn's adenoma, and Cushing's syndrome (Table 4). The perioperative complications are listed in Table 5. There were no bowel or major vascular injuries in both groups. 


\section{DISCUSSION}

Partial adrenalectomy (PA) was first introduced as a method of preserving the adrenal cortical tissue. It avoids the need for life-long hormone replacement. PA is particularly meaningful in patients with bilateral involvement, those with a solitary adrenal gland, as well as those with hereditary adrenal diseases such as multiple endocrine neoplasia (MEN-2) and von Hippel-Landau disease. Indeed, steroid replacement therapy is unavoidable following bilateral TA. This may require dose adjustments during stressful events and increase a patient's risk of overtreatment and its subsequent damaging effects on bone and other organs. ${ }^{13,14}$ Nakada et al. $^{3}$ have found that patients with unilateral adrenalectomy do not respond as robustly to stressful situations as do normal controls. Furthermore, if the remaining adrenal gland requires future surgical therapy or suffers functional impairment, the patient may lose his/her remaining source of steroid production. Therefore, preserving the maximal amount of normal adrenal cortex can leave patients with adequate hormonal reserve and avoid the need for long-term steroid therapy. Based on this, physicians have begun to extend partial excision to sporadic unilateral tumors. ${ }^{14}$ During the last 2 decades, the concept of LPA has been increasingly adopted for its safety and feasibility in cases of pheochromocytomas, Cushing's syndrome, and Conn's adenomas. ${ }^{15-20}$

Walz et al. ${ }^{21}$ reported on their experience with 100 LPAs. There were no differences between the TA and PA groups with regard to tumor size, operating time, or blood loss. They used several modalities including electrocautery, ultrasonic energy, or clips for tumor resection. In all patients with PA, biochemical healing was proven by no evidence of recurrence after 51 months of follow-up.

Additionally, Jeschke et al. ${ }^{12}$ reported on 13 patients who underwent transperitoneal LPA for Conn's adenoma. They applied a combination of preliminary bipolar coagulation and endoshears to excise the tumor with a 2- to 3-mm margin under intraoperative ultrasound guidance. Contrary to Walz et al., ${ }^{21}$ main adrenal vein was preserved to maintain the circulation of the remaining adrenal gland. All patients had normal blood pressure and aldosterone level at a mean follow-up of 39 months, with no recurrences.

We performed LPA in cases of pheochromocytoma, NAA, primary aldosteronism, Cushing's syndrome, adrenal cyst, and so on. In contrast, we did not try LPA in primary adrenocortical carcinoma or metastatic carcinoma with exception of one incidental case. We preferred LPA whenever the tumor was located on a periphery of the affected adrenal limb. In our study, there were comparable outcomes between LPA and LTA with regard to operating time, estimated blood loss, complication rate, and long-term functional result. No local recurrences were found during follow-up for a maximum of 125 months. Biochemical laboratory data have been normalized after operation in hormonally active patients. At the mean follow-up of 49.2 \pm 39.5 months, most of the Conn's adenoma patients remained asymptomatic, with stabilized blood pressure. However, about $25 \%$ of the patients had sustained hypertension and still required eventually reduced antihypertensive medication following surgery in both groups. It is possible that these patients had a superimposed essential hypertension. Therefore, it is important to counsel patients that their blood pressure may not be normalized after surgery. In addition, the plasma or 24-hr urinary metanephrine level as well as their relevant symptoms normalized postoperatively in all patients with pheochromocytoma. In cases of Cushing's syndrome, all patients received a corticosteroid medication perioperatively. However, the drug could be ceased within 6 months in all patients after the LPA.

When performing LPA for a pheochromocytoma, the surgeon must recognize the increased risk of hypertensive crisis. The reason for this is not only because there is a higher possibility of tumor manipulation during LPA dissection than there is in LTA, but also because the adrenal vein is not routinely ligated in LPA cases. According to our experience, early isolation of the main adrenal vein in cases of large tumor is an important key step for LPA, because it allows the surgeon to immediately ligate this vein in the setting of intraoperative, uncontrollable hypertensive crisis. In contrast, in cases of peripherally located small pheochromocytoma, we could preserve the adrenal vein without any difficulty.

The adrenal gland is one of the most highly vascular organs in the human body. Therefore, LPA has always held a significant risk of perioperative bleeding. Various techniques for PA, as well as additional hemostatic modalities, have been reported. Commonly used reliable devices for adrenal cutting and hemostasis are electrocautery and ultrasonic energy, respectively. Some studies have demonstrated that the ultrasonic scalpel is efficacious in bleeding control when used during PA. ${ }^{22-25}$ Additional methods to improve hemostasis include the use of 
argon beam laser or bipolar coagulation, fibrin glue, and clips. ${ }^{15}$ The vascular stapler was also efficacious in hemostasis during LPA. ${ }^{26}$ However, the authors found that endo GIA stapler could not always be applied because of the variable tumor location. We performed minimal dissection of the tissues around the normal adrenal to guarantee collateral circulation in the remnant adrenal tissue. After mobilizing an adequate part of the adrenal gland, we divided the adrenal tissue from the side of the normal adrenal limb using Hem-o-lok clips (Weck Surgical Instruments, Teleflex Medical, Durham, NC, USA). These clips allowed us to achieve good hemostasis (Fig. 1). In addition, we usually applied fibrin glue and Surgicel (Ethicon Inc., Cincinnati, OH, USA) along the resection margin to encourage hemostasis. In fact, we had used various methods such as monopolar electrocautery, bipolar energy device or even direct adrenal suture to control bleeding on early cases, but we found that using Hem-o-lok clips was the most effective method for hemostasis.

Surgeons are increasingly using intraoperative ultrasound during LPA. ${ }^{27}$ Intraoperative ultrasound has also been shown to be useful for the identification of the lesion during laparoscopic surgery of extra-adrenal pheochromocytomas. ${ }^{28}$ In these cases, intraoperative ultrasonography serves to search for additional lesions not seen on preoperative imaging study, shows the main adrenal vein as well as the tumor vascularity, and assesses the possibility of PA, especially if the adrenal tumor is large or in a vague location to resect partially. Unfortunately, we were not able to use the device because our center did not have an available intracorporeal ultrasound probe for the first several years of this study. Regardless, there were no difficulties in detecting the mass intraoperatively due to high-resolution imaging tools such as CT or MRI.

\section{CONCLUSIONS}

Our study reveals that LPA is a safe, technically feasible procedure. It is useful not only for biochemically active adrenal tumors such as Conn's adenoma, pheochromocytoma, or Cushing's syndrome, but also for hormonally nonfunctioning adrenal tumors. We demonstrated that LPA has 2 major advantages such as shorter operation time and more preservation of functioning adrenal gland compared to those of LTA. This includes cases in which the adrenal tumor is unilateral or bilateral, in both inert and biochemically active tumors. Our data also show that functional and oncological outcomes of LPA are comparable to those of LTA. Further studies are needed to determine the amount of remnant adrenal tissue required to provide adequate hormonal reserve. In addition, future researches will determine which technique is the most ideal for a guarantee of adequate bleeding control, and in what setting each procedure is indicated with regard to tumor size and location.

\section{CONFLICT OF INTEREST}

The authors claim no conflicts of interest.

\section{REFERENCES}

1. Telenius-Berg M, Ponder MA, Berg B, Ponder BA, Werner S. Quality of life after bilateral adrenalectomy in MEN 2. Henry Ford Hosp Med J 1989;37:160-3.

2. Lucon AM, Mendonça BB, Domenice S, Chambô JL, Wajchemberg BL, Arap S. Adrenal autografts following bilateral adrenalectomy. J Urol 1993;149:977-9.

3. Nakada T, Kubota Y, Sasagawa I, Yagisawa T, Watanabe M, Ishigooka M. Therapeutic outcome of primary aldosteronism: adrenalectomy versus enucleation of aldosterone-producing adenoma. J Urol 1995;153:1775-80.

4. van Heerden JA, Sizemore GW, Carney JA, Brennan MD, Sheps SG. Bilateral subtotal adrenal resection for bilateral pheochromocytomas in multiple endocrine neoplasia, type IIa: a case report. Surgery 1985;98:363-6.

5. Bornstein SR. Predisposing factors for adrenal insufficiency. N Engl J Med 2009;360:2328-39.

6. Marx JA, editor. Rosen's emergency medicine concepts and clinical practice. 6th ed. Philadelphia (PA): Mosby; 2006.

7. Sasagawa I, Suzuki Y, Itoh K, Izumi T, Miura M, Suzuki H, et al. Posterior retroperitoneoscopic partial adrenalectomy: clinical experience in 47 procedures. Eur Urol 2003;43:381-5.

8. Roukounakis N, Dimas S, Kafetzis I, Bethanis S, Gatsulis N, Kostas $\mathrm{H}$, et al. Is preservation of the adrenal vein mandatory in laparoscopic adrenal-sparing surgery? JSLS 2007;11:215-8.

9. Castillo OA, Vitagliano G, Cortes O, Kerkebe M, Pinto I, Arellano L. Bilateral laparoscopic adrenalectomy. J Endourol 2007;21:1053-8.

10. Diner EK, Franks ME, Behari A, Linehan WM, Walther MM. Partial adrenalectomy: the National Cancer Institute experience. Urology 2005;66:19-23.

11. Edström E, Gröndal S, Norström F, Palmér M, Svensson KA, Widell $\mathrm{H}$, et al. Long term experience after subtotal adrenalectomy for multiple endocrine neoplasia type IIa. Eur J Surg 1999;165:431-5.

12. Jeschke K, Janetschek G, Peschel R, Schellander L, Bartsch 
G, Henning K. Laparoscopic partial adrenalectomy in patients with aldosterone-producing adenomas: indications, technique, and results. Urology 2003;61:69-72.

13. Birnbaum J, Giuliano A, Van Herle AJ. Partial adrenalectomy for pheochromocytoma with maintenance of adrenocortical function. J Clin Endocrinol Metab 1989;69:1078-81.

14. Gross MD, Shapiro B, Freitas JE, Meyers L, Francis I, Thompson NW, et al. Clinical significance of the solitary functioning adrenal gland. J Nucl Med 1991;32:1882-7.

15. Janetschek G, Finkenstedt G, Gasser R, Waibel UG, Peschel $\mathrm{R}$, Bartsch G, et al. Laparoscopic surgery for pheochromocytoma: adrenalectomy, partial resection, excision of paragangliomas. J Urol 1998;160:330-4.

16. Pautler SE, Choyke PL, Pavlovich CP, Daryanani K, Walther MM. Intraoperative ultrasound aids in dissection during laparoscopic partial adrenalectomy. J Urol 2002;168:1352-5.

17. Kok KY, Yapp SK. Laparoscopic adrenal-sparing surgery for primary hyperaldosteronism due to aldosterone-producing adenoma. Surg Endosc 2002;16:108-11.

18. Ikeda Y, Takami H, Niimi M, Kan S, Sasaki Y, Takayama J. Laparoscopic partial or cortical-sparing adrenalectomy by dividing the adrenal central vein. Surg Endosc 2001;15:74750 .

19. Simone G, Anceschi U, Tuderti G, Misuraca L, Celia A, De Concilio B, et al. Robot-assisted partial adrenalectomy for the treatment of Conn's syndrome: surgical technique, and perioperative and functional outcomes. Eur Urol 2019;75: 811-6.

20. He HC, Dai J, Shen ZJ, Zhu Y, Sun FK, Shao Y, et al. Retroperitoneal adrenal-sparing surgery for the treatment of Cushing's syndrome caused by adrenocortical adenoma: 8-year experience with 87 patients. World J Surg 2012;36: 1182-8.
21. Walz MK, Peitgen K, Diesing D, Petersenn S, Janssen OE, Philipp T, et al. Partial versus total adrenalectomy by the posterior retroperitoneoscopic approach: early and long-term results of 325 consecutive procedures in primary adrenal neoplasias. World J Surg 2004;28:1323-9.

22. Al-Sobhi S, Peschel R, Zihak C, Bartsch G, Neumann H, Janetschek G. Laparoscopic partial adrenalectomy for recurrent pheochromocytoma after open partial adrenalectomy in von Hippel-Lindau disease. J Endourol 2002;16:171-4.

23. Walther MM, Herring J, Choyke PL, Linehan WM. Laparoscopic partial adrenalectomy in patients with hereditary forms of pheochromocytoma. J Urol 2000;164:14-7.

24. Yuge K, Miyajima A, Hasegawa M, Miyazaki Y, Maeda T, Takeda $\mathrm{T}$, et al. Initial experience of transumbilical laparoendoscopic single-site surgery of partial adrenalectomy in patient with aldosterone-producing adenoma. BMC Urol 2010;10:19.

25. Suzuki K, Sugiyama T, Saisu K, Ushiyama T, Fujita K. Retroperitoneoscopic partial adrenalectomy for aldosterone-producing adenoma using an ultrasonically activated scalpel. Br J Urol 1998;82:138-9.

26. Sasagawa I, Suzuki H, Tateno T, Izumi T, Shoji N, Nakada $\mathrm{T}$. Retroperitoneoscopic partial adrenalectomy using an endoscopic stapling device in patients with adrenal tumor. Urol Int 1998;61:101-3.

27. Heniford BT, Iannitti DA, Hale J, Gagner M. The role of intraoperative ultrasonography during laparoscopic adrenalectomy. Surgery 1997;122:1068-73.

28. Hwang J, Shoaf G, Uchio EM, Watson J, Pacak K, Linehan $\mathrm{WM}$, et al. Laparoscopic management of extra-adrenal pheochromocytoma. J Urol 2004;171:72-6. 